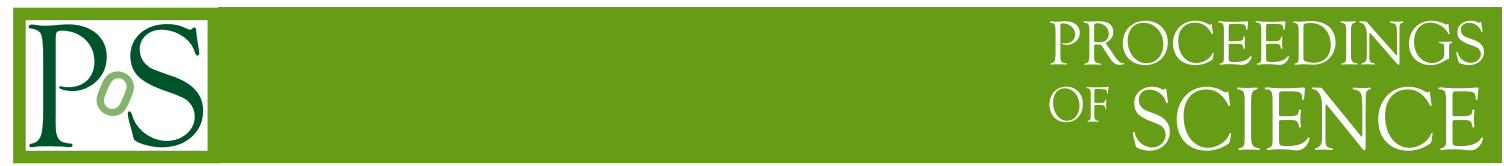

ROM2F/2008/23, DESY 08-139, SFB/CPP-08-78, LTH806, HU-EP-08/41

\title{
Scaling and chiral extrapolation of pion mass and decay constant with maximally twisted mass QCD
}

\author{
Petros Dimopoulos, Roberto Frezzotti \\ Dip. di Fisica, Università di Roma "Tor Vergata" \\ Via della Ricerca Scientifica 1, 00133 Rome, Italy \\ E-mail: \{dimopoulos, frezzotti\} droma2.infn.it \\ Gregorio Herdoiza, Karl Jansen \\ DESY \\ Platanenallee 6, 15738 Zeuthen, Germany \\ E-mail: \{Gregorio.Herdoiza, Karl.Jansen\}@desy.de
}

Chris Michael

Theoretical Physics Division, Department of Mathematical Sciences, University of Liverpool

Liverpool L69 3BX, UK

E-mail: c.michaeleliverpool.ac.uk

\section{Carsten Urbach*}

Humboldt-Universität zu Berlin, Institut für Physik,

Newtonstr. 15, 12489 Berlin, Germany

E-mail: Carsten.Urbach@physik.hu-berlin.de

We present an update of the results for pion mass and pion decay constant as obtained by the ETM collaboration in large scale simulations with maximally twisted mass fermions and two mass degenerate flavours of light quarks. We discuss the continuum, chiral and infinite volume extrapolation of these quantities as well as the extraction of low energy constants, and investigate possible systematic uncertainties.

The XXVI International Symposium on Lattice Field Theory

July 14-192008

Williamsburg, Virginia, USA

\footnotetext{
*Speaker.
} 


\begin{tabular}{lccccccc}
\hline \hline Ensemble & $L^{3} \times T$ & $\beta$ & $a \mu_{q}$ & $\kappa$ & $\tau_{\text {int }}(P)$ & $\tau_{\text {int }}\left(a m_{\mathrm{PS}}\right)$ & $\tau$ \\
\hline \hline$B_{1}$ & $24^{3} \times 48$ & 3.9 & 0.0040 & 0.160856 & $47(15)$ & $7(1)$ & 0.5 \\
$B_{2}$ & & & 0.0064 & & $23(7)$ & $17(4)$ & 0.5 \\
$B_{3}$ & & 0.0085 & & $13(3)$ & $10(2)$ & 0.5 \\
$B_{4}$ & & & 0.0100 & & $15(4)$ & $7(2)$ & 0.5 \\
$B_{5}$ & & 0.0150 & & $30(8)$ & $20(6)$ & 0.5 \\
$B_{6}$ & $32^{3} \times 64$ & 3.9 & 0.0040 & 0.160856 & $37(11)$ & $2.8(3)$ & 0.5 \\
$B_{7}$ & & & 0.0030 & & $51(19)$ & $7(1)$ & 1.0 \\
\hline \hline
\end{tabular}

Table 1: Update of the ensembles produced with $\beta=3.9$ by the ETM collaboration. For the other $\beta$-values see table 1 of Ref. [2]. We give the lattice volume $L^{3} \times T$, the twisted mass parameter $a \mu_{q}$, the hopping parameter $\kappa=1 /\left(8+2 a m_{0}\right)$ and the trajectory length $\tau$. In addition we provide values for the integrated autocorrelation time of two typical quantities, the plaquette $P$ and the pseudo scalar mass $a m_{\mathrm{PS}}$, in units of $\tau=0.5$.

\section{Introduction}

We present an update of the $n_{f}=2$ results obtained by the European Twisted Mass collaboration (ETMC) for the pseudo scalar mass and decay constant. A good understanding of continuum, thermodynamic and chiral limits is essential in order to obtain reliable results, which can eventually be compared to experiment. The physics of the light pseudo scalar meson is a prime example for investigating these extrapolations, because its mass and decay constant can be obtained with high precision in lattice simulations and chiral perturbation theory $(\chi \mathrm{PT})$ is best understood for those two quantities. In addition, such an investigation allows to extract other quantities of phenomenological interest, such as low energy constants and quark masses.

First results for the pseudo scalar mass and decay constant obtained for the large scale simulations of the ETM collaboration can be found in Refs. [1-4].

ETMC has generated large sets of gauge configurations for different values of the coupling constant $(\beta=3.8, a \sim 0.1 \mathrm{fm} ; \beta=3.9, a \sim 0.085 \mathrm{fm} ; \beta=4.05, a \sim 0.065 \mathrm{fm}$ ), for various volumes $(2.1-2.8 \mathrm{fm})$ and a number of bare quark masses corresponding to pseudo scalar meson masses ranging from $\sim 260$ to $\sim 700 \mathrm{MeV}$. The list of ensembles at $\beta=3.9$ can be found in table 1 , which contains the newly generated ensemble $B_{7}$ corresponding to a pseudo scalar meson mass of about $m_{\mathrm{PS}} \sim 265 \mathrm{MeV}$. For the other $\beta$-values we refer to table 1 of Ref. [2].

In the gauge sector we employ the so-called tree-level Symanzik improved gauge action (tlSym) [5]. The fermionic action for two flavours of maximally twisted, mass degenerate quarks in the so called twisted basis $[6,7]$ reads

$$
S_{\mathrm{tm}}=a^{4} \sum_{x}\left\{\bar{\chi}(x)\left[D[U]+m_{0}+i \mu_{q} \gamma_{5} \tau^{3}\right] \chi(x)\right\}
$$

where $m_{0}$ is the untwisted bare quark mass tuned to its critical value $m_{\text {crit }}, \mu_{q}$ is the bare twisted quark mass, $\tau^{3}$ is the third Pauli matrix acting in flavour space and $D[U]$ is the Wilson-Dirac operator.

At maximal twist, i.e. $m_{0}=m_{\text {crit }}$, physical observables are automatically $\mathscr{O}(a)$ improved without the need to determine any action or operator specific improvement coefficients [7] (for a re- 


\begin{tabular}{lrrr}
\hline \hline & $\beta$ & $a \mu_{q}$ & $R_{O}$ \\
\hline \hline$a f_{\mathrm{PS}}$ & 3.90 & 0.004 & $0.04(06)$ \\
& 4.05 & 0.003 & $-0.03(06)$ \\
$a m_{\mathrm{V}}$ & 3.90 & 0.004 & $0.02(07)$ \\
& 4.05 & 0.003 & $-0.10(11)$ \\
$a f_{\mathrm{V}}$ & 3.90 & 0.004 & $-0.07(18)$ \\
& 4.05 & 0.003 & $-0.31(29)$ \\
$a m_{\Delta}$ & 3.90 & 0.004 & $0.022(29)$ \\
& 4.05 & 0.003 & $-0.004(45)$ \\
\hline \hline
\end{tabular}

Table 2: Comparison of some selected quantities for which an isospin splitting can occur for twisted mass fermions. $R_{O}$ denotes the measured relative size of the splitting.

view see Ref. [8]). With this being the main advantage, one drawback of maximally twisted mass fermions is that flavour symmetry is broken explicitly at finite value of the lattice spacing, which amounts to $\mathscr{O}\left(a^{2}\right)$ effects in physical observables, as will be discussed later. Note that in the following we shall refer to the charged pseudo scalar meson mass as $m_{\mathrm{PS}}$ or $m_{\mathrm{PS}}^{ \pm}$and to the neutral one as $m_{\mathrm{PS}}^{0}$.

For details on the set-up, tuning to maximal twist and the analysis methods of the ETM collaboration we refer to Refs. [1, 2, 4]. Recent results for light quark masses and decay constants, the light baryon spectrum and the $\eta^{\prime}$ meson are available in Refs. [9, 10] and Ref. [11], respectively. We shall only consider the ensembles at $\beta=3.90$ ( $B$-ensembles) and $\beta=4.05$ ( $C$-ensembles, see table 1 of Ref. [2]) in this proceeding contribution, because tuning to maximal twist at $\beta=3.8$ was not sufficiently accurate at the lowest quark mass values for the observables considered here.

\section{Results}

\section{Flavour Breaking Effects}

Flavour breaking effects have been investigated by ETMC for several quantities. In figure 1(a) we plot $r_{0}^{2}\left(\left(m_{\mathrm{PS}}^{ \pm}\right)^{2}-\left(m_{\mathrm{PS}}^{0}\right)^{2}\right)$ as a function of $\left(a / r_{0}\right)^{2}$. It is visible that mass splitting of the charged to neutral pseudo scalar meson is large. However, the measured splittings are compatible with being an $\mathscr{O}\left(a^{2}\right)$ effect, as expected, and they vanish towards the continuum limit.

All other possible splittings investigated so far are compatible with zero. In table 2 we have compiled the relative difference $R_{O}=\left(O-O^{\prime}\right) / O$ for some selected simulation points and observables $O$. Here $O\left(O^{\prime}\right)$ denotes the charged (neutral) quantity for mesons and $\Delta^{+}\left(\Delta^{++}\right)$for baryons. The values of $R_{O}$ are well compatible with zero for all observables $O$ besides the pion mass. However, some quantities, like the vector meson decay constant $f_{V}$, are rather noisy, making definite conclusions difficult. These results are compatible with a theoretical investigation using the Symanzik effective Langrangian [12].

\section{SU(2) $\chi$ PT Fits}

As the details of finite size corrections for $m_{\mathrm{PS}}$ and $f_{\mathrm{PS}}$ were discussed in Ref. [2] and the issue 


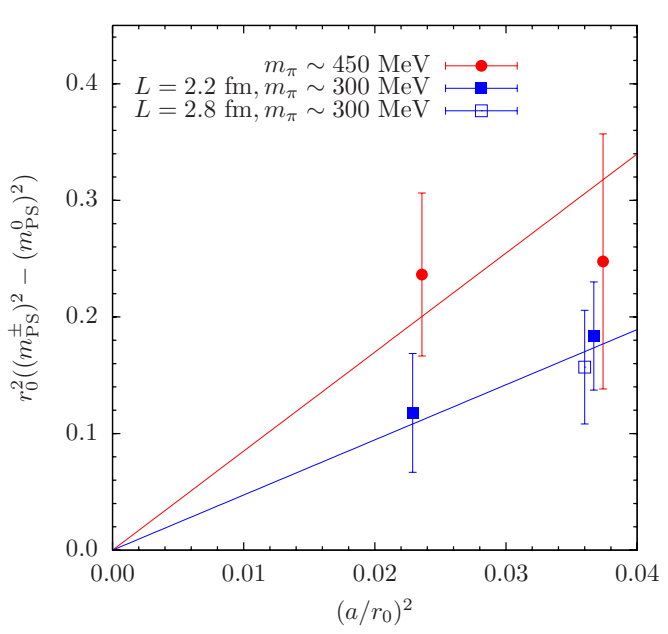

(a)

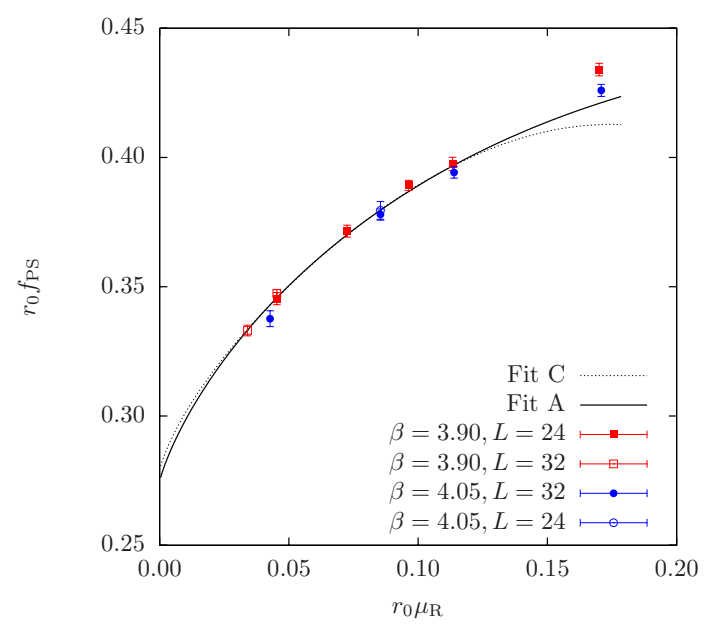

(b)

Figure 1: (a) Mass splitting $r_{0}^{2}\left(\left(m_{\mathrm{PS}}^{ \pm}\right)^{2}-\left(m_{\mathrm{PS}}^{0}\right)^{2}\right)$ as a function of $\left(a / r_{0}\right)^{2}$. (b) Data for $r_{0} f_{\mathrm{PS}}$ as a function of $r_{0} \mu_{R}$ for $\beta=3.90$ and $\beta=4.05$ and Fits $\mathrm{A}$ and $\mathrm{C}$.

of continuum extrapolation in fixed and finite volume for selected quantities in Refs. [2, 3], we shall summarise here only the main results:

1. finite size effects in $f_{\mathrm{PS}}$ and $m_{\mathrm{PS}}$ can be described using chiral perturbation theory in the form of the resummed Lüscher formula as described in Ref. [13]. We denote the corresponding correction factors with $K_{f, m}^{\mathrm{CDH}}$, which depend among others on the low energy constants $\Lambda_{1-4}$.

2. within our current statistical precision lattice artifacts appear to be negligible, in particular for $m_{\mathrm{PS}}, f_{\mathrm{PS}}$, the quantities we consider here. In fixed volume and at fixed value of $r_{0} m_{\mathrm{PS}}$ the results for $r_{0} f_{\mathrm{PS}}$ at $\beta=3.9$ and $\beta=4.05$ are always compatible with each other within our small statistical errors, and hence a constant extrapolation to the continuum limit seems justified. When we include also a linear term in $\left(a / r_{0}\right)^{2}$ in the extrapolation, a least square fit determines a value for the slope that is zero within errors. However, we include this effect into our systematic uncertainties.

We shall now present the results for a combined chiral, thermodynamic and continuum extrapolation of $m_{\mathrm{PS}}$ and $f_{\mathrm{PS}}$ for the two $\beta$-values $\beta=3.9$ and $\beta=4.05$. What we present here will extend the results given in Refs. [2,3] by incorporating chirally extrapolated data for the renormalisation constant $Z_{\mathrm{P}}$ and the Sommer parameter $r_{0} / a$ into the fit. Details on the computation of $Z_{\mathrm{P}}$ (using the RIMOM) and $r_{0} / a$ can be found in Refs. $[14,4]$.

We perform combined fits to our data for $f_{\mathrm{PS}}, m_{\mathrm{PS}}, r_{0} / a$ and $Z_{P}$ at the two values of $\beta$ with the formulae:

$$
\begin{aligned}
r_{0} f_{\mathrm{PS}} & =r_{0} f_{0}\left[1-2 \xi \log \left(\frac{\chi_{\mu}}{\Lambda_{4}^{2}}\right)+D_{f_{\mathrm{PS}}}\left(a / r_{0}\right)^{2}+T_{f}^{\mathrm{NNLO}}\right] K_{f}^{\mathrm{CDH}}(L), \\
\left(r_{0} m_{\mathrm{PS}}\right)^{2} & =\chi_{\mu} r_{0}^{2}\left[1+\xi \log \left(\frac{\chi_{\mu}}{\Lambda_{3}^{2}}\right)+D_{m_{\mathrm{PS}}}\left(a / r_{0}\right)^{2}+T_{m}^{\mathrm{NNLO}}\right] K_{m}^{\mathrm{CDH}}(L)^{2},
\end{aligned}
$$




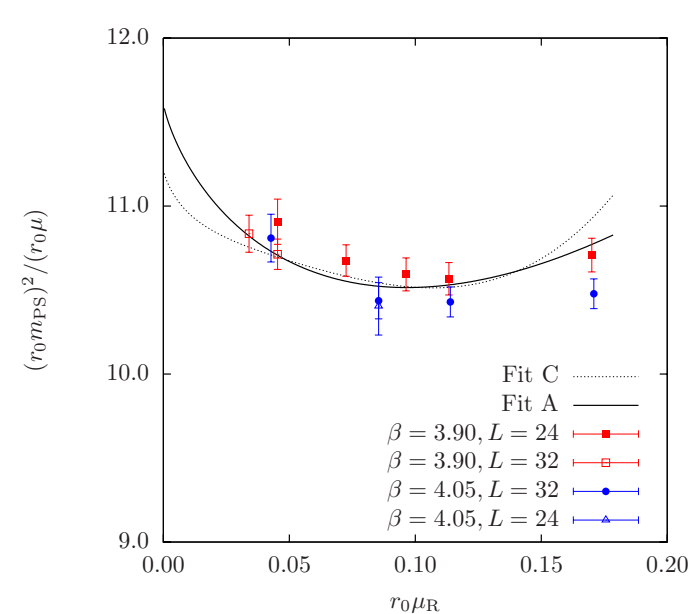

(a)

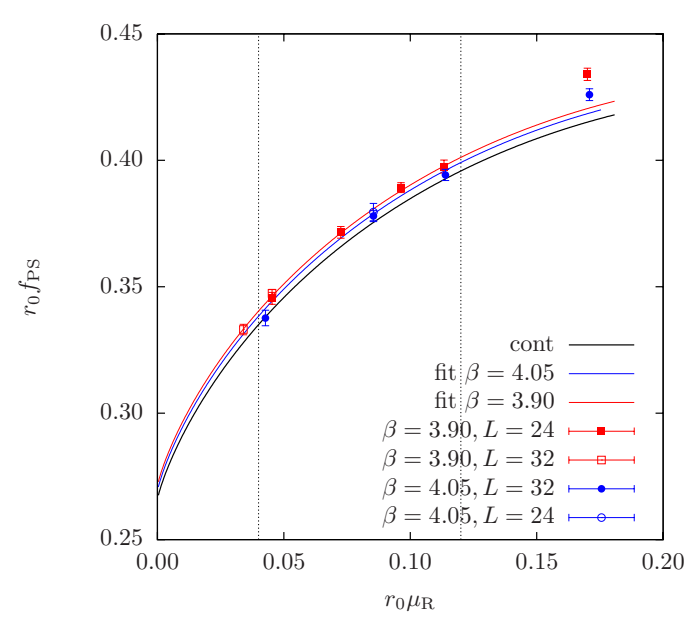

(b)

Figure 2: (a) Data for $\left(r_{0} m_{\mathrm{PS}}\right)^{2} / r_{0} \mu_{R}$ as a function of $r_{0} \mu_{r}$ and Fits A and C. (b) Data for $r_{0} f_{\mathrm{PS}}$ as a function of $r_{0} \mu_{R}$ and resulting curves of Fit $\mathrm{B}$. The vertical lines indicate the fit range.

with $\xi \equiv 2 B_{0} \mu_{R} /\left(4 \pi f_{0}\right)^{2}, \chi_{\mu} \equiv 2 B_{0} \mu_{R}, \mu_{R} \equiv \mu_{q} / Z_{\mathrm{P}}, f_{0} \equiv \sqrt{2} F_{0} . T_{m, f}^{\mathrm{NNO}}$ denote the continuum NNLO terms [15], which depend on $\Lambda_{1-4}$ and $k_{M}$ and $k_{F}$, and $K_{m, f}^{\mathrm{CDH}}(L)$ the finite size corrections [13]. Based on the form of the Symanzik expansion in the small quark mass region, we parametrise in eq. (2.1) the leading cut-off effects by the two coefficients $D_{f_{\mathrm{PS}}, m_{\mathrm{PS}}}$.

At NLO, i.e. setting $T_{m, f}^{\mathrm{NNLO}} \equiv 0$, and neglecting finite size corrections for the moment, there are the following free parameters to be fitted to the data for $a f_{\mathrm{PS}}, a m_{\mathrm{PS}}, r_{0} / a$ and $Z_{\mathrm{P}}$ :

$$
r_{0} f_{0}, r_{0} B_{0}, r_{0} \Lambda_{3}, r_{0} \Lambda_{4},\left\{r_{0} / a\right\}_{\beta},\left\{Z_{\mathrm{P}}\right\}_{\beta}, D_{m_{\mathrm{PS}}}, D_{f_{\mathrm{PS}}},
$$

where we indicate with the notation $\{\ldots\}_{\beta}$ that there is one parameter for each value $a$.

Finite size effects are corrected for by using the asymptotic formulae from $\mathrm{CDH}$, which is consistently included in the fit. However, $K_{f, m}^{\mathrm{CDH}}$ depend on more parameters $\left(\Lambda_{1,2}, \tilde{r}_{1-4}\right)$. Those we do not fit, but set them to the values suggested in Ref. [16] using the physical value of $r_{0}$ as determined from the fit. This appears to be justified, since we are able to describe our measured finite size effects.

When including NNLO terms into the fit there are four additional parameters to be determined. We cannot fit them to the data, because the fits become unstable. In order to be still able to estimate systematic uncertainties from NNLO contributions, we include priors for $r_{0} \Lambda_{1,2}, k_{M}, k_{F}$ into the fit when NNLO terms are included. As priors we use for $k_{M, F}=0 \pm 1$ and for $r_{0} \Lambda_{1,2}$ the values given in Ref. [16].

Our fit procedure can be viewed as first extrapolating the data to the continuum limit and use continuum chiral perturbation theory afterwards for the chiral and infinite volume extrapolations. For this reason we do not expect any influence of the neutral pseudo scalar meson on the finite size effects: in the continuum all three pseudo scalar mesons are degenerate. Note that setting $D_{m_{\mathrm{PS}}, f_{\mathrm{PS}}} \equiv 0$ corresponds to a constant continuum extrapolation. Using the boostrap method to estimate the statistical uncertainties, we performed the following fits

1. Fit A: NLO continuum $\chi \mathrm{PT}, T_{m, f}^{\mathrm{NNLO}} \equiv 0, D_{m_{\mathrm{PS}}, f_{\mathrm{PS}}} \equiv 0$, ensembles $B_{1,2,3,4,6}$ and $C_{1,2,3,5}$ 
2. Fit B: NLO continuum $\chi \mathrm{PT}, T_{m, f}^{\mathrm{NNLO}} \equiv 0, D_{m_{\mathrm{PS}}, f_{\mathrm{PS}}}$ fitted, ensembles $B_{1,2,3,4,6}$ and $C_{1,2,3,5}$

3. Fit C: NNLO continuum $\chi \mathrm{PT}, D_{m_{\mathrm{PS}}, f_{\mathrm{PS}}} \equiv 0$, ensembles $B_{1,2,3,4,6}$ and $C_{1,2,3,5}$

4. Fit D: like Fit A, but ensembles $B_{5}$ and $C_{4}$ added

5. Fit E: like Fit A, but ensemble $B_{7}$ added

Using the fitted parameters we can then determine low energy constants like $\bar{\ell}_{3,4}$, the chiral condensate $\Sigma$ and the pseudo scalar decay constant in the chiral limit $f_{0}$.

\section{Discussion}

The fit results are summarised in table 3. In terms of $\chi^{2} /$ dof the Fits A, B, C and E provide a good description of the data with $\chi^{2} /$ dof $\sim 1$, whereas Fit $\mathrm{D}$, which includes simulation points with $m_{\mathrm{PS}} \sim 600 \mathrm{MeV}$, has significantly larger $\chi^{2} / \mathrm{dof}$. We conclude from this that $\chi \mathrm{PT}$ is not applicable for values of $m_{\mathrm{PS}}>500 \mathrm{MeV}$.

To the contrary, including ensemble $B_{7}$ as in Fit E, and hence extending the fit-range to a value of $m_{\mathrm{PS}} \sim 265 \mathrm{MeV}$ reveals completely consistent results with Fit A. This result makes us confident that the extrapolation to the physical point is trustworthy.

Including lattice artifacts in the fit (Fit B) does change rather little as compared to Fit A, and the coefficients $D_{m_{\mathrm{PS}}, f_{\mathrm{PS}}}$ are compatible with zero, while the value of $\chi^{2} /$ dof is not significantly reduced: the differences between the results at $\beta=3.9$ and $\beta=4.05$ can be explained with the variance observed in $r_{0} / a$ and $Z_{\mathrm{P}}$. Hence, we have to reduce our (already small) statistical errors even further to resolve lattice artifacts in $f_{\mathrm{PS}}$ and $m_{\mathrm{PS}}$, indicating small lattice artifacts in those two quantities. When NNLO terms are included in the Fit (Fit C), the most significant difference compared to Fit $\mathrm{A}$ is observed for $\bar{\ell}_{3}$. Though this effect is not significant, we include it as a systematic error in our final results.

These findings are visualised in figures 1(b), 2(a) and 2(b). In figure 1(b) we plot $r_{0} f_{\mathrm{PS}}$ as a function of the renormalised quark mass $r_{0} \mu_{R}$ comparing Fits A and C. In the range $0.04 \leq$ $r_{0} \mu_{R} \leq 0.12$ the two fits agree remarkably well, while for $r_{0} \mu_{R}>0.12$ both fail to describe the data. Note that we might be seeing lattice artifacts of the order $a^{2} \mu_{q}^{2}$ at these large masses, which would explain the difference between the results at $\beta=3.9$ and $\beta=4.05$. Similar conclusions can be drawn from figure 2(a), where we plot $\left(r_{0} m_{\mathrm{PS}}\right)^{2} /\left(r_{0} \mu_{R}\right)$ as a function of the renormalised quark mass.

In figure 2(b) we show the result of Fit B for $r_{0} f_{\mathrm{PS}}$. The three curves correspond to the fitted curve at $\beta=3.9$ (red), the fitted curve at $\beta=4.05$ (blue) and to the continuum curve (black). The differences between the three curves are rather small, reflecting the result that $D_{m_{\mathrm{PS}}, f_{\mathrm{PS}}}$ are zero within errors.

\section{Conclusion and Outlook}

We have presented an update of the ETMC results for $f_{\mathrm{PS}}$ and $m_{\mathrm{PS}}$ and their continuum, thermodynamic and chiral extrapolations. The main difference to the previous analysis in Ref. [2, 3] is a new simulation point at $\beta=3.9$ and the inclusion of $r_{0} / a$ and $Z_{\mathrm{P}}$ data into the fit. The main results are summarised with $\bar{\ell}_{3}=3.42(8)(10)(27), \bar{\ell}_{4}=4.59(4)(2)(13), \Sigma^{\overline{\mathrm{MS}}}(2 \mathrm{GeV})=$ 


\begin{tabular}{lrrrrr}
\hline \hline & Fit A & Fit B & Fit C & Fit D & Fit E \\
\hline$\overline{\bar{\ell}}_{3}$ & $3.42(8)$ & $3.52(8)$ & $3.15(19)$ & $3.55(5)$ & $3.41(7)$ \\
$\bar{\ell}_{4}$ & $4.59(4)$ & $4.61(4)$ & $4.72(12)$ & $4.72(2)$ & $4.60(3)$ \\
$\Sigma^{\overline{\mathrm{MS}}}(2 \mathrm{GeV})\left[\mathrm{MeV}^{3}\right]$ & $(-267(2))^{3}$ & $(-276(5))^{3}$ & $(-263(2))^{3}$ & $(-269(1))^{3}$ & $(-267(1))^{3}$ \\
$f_{0}[\mathrm{MeV}]$ & $121.66(7)$ & $121.6(1)$ & $121.7(3)$ & $121.39(5)$ & $121.64(7)$ \\
$f_{\pi} / f_{0}$ & $1.0743(7)$ & $1.0746(9)$ & $1.0739(23)$ & $1.0767(4)$ & $1.0745(6)$ \\
$D_{m_{\mathrm{PS}}}$ & - & $-1.4(1.3)$ & - & - & - \\
$D_{f_{\mathrm{PS}}}$ & - & $+0.58(69)$ & - & - & - \\
$\chi^{2} /$ dof & $17.7 / 14$ & $12.9 / 12$ & $15.3 / 14$ & $46.7 / 18$ & $18.6 / 16$ \\
\hline \hline
\end{tabular}

Table 3: Summary of fit results.

$(-267(2)(9)(4) \mathrm{MeV})^{3}$ and $f_{\pi} / f_{0}=1.0743(7)(3)(4)$. The first error is statistical, the second estimates residual lattice artifacts and the third effects from NNLO $\chi$ PT. In addition we have presented results indicating that flavour breaking effects are zero within the statistical accuracy, with the exception of the neutral pseudo scalar meson mass.

We thank all members of ETMC for the most enjoyable collaboration. This work has been supported in part by the DFG Sonderforschungsbereich/ Transregio SFB/TR9-03.

\section{References}

[1] ETM Collaboration, P. Boucaud et al., Phys. Lett. B650, 304 (2007), arXiv: hep-lat / 0701012.

[2] ETM Collaboration, C. Urbach, PoS LAT2007, 022 (2007), arXiv: 0710.1517 [hep-lat].

[3] ETM Collaboration, P. Dimopoulos, R. Frezzotti, G. Herdoiza, C. Urbach and U. Wenger, PoS LAT2007, 102 (2007), arXiv: 0710.2498 [hep-lat] .

[4] ETM Collaboration, P. Boucaud et al., arXiv:0803.0224 [hep-lat ].

[5] P. Weisz, Nucl. Phys. B212, 1 (1983).

[6] ALPHA Collaboration, R. Frezzotti, P. A. Grassi, S. Sint and P. Weisz, JHEP 08, 058 (2001), hep-lat/0101001.

[7] R. Frezzotti and G. C. Rossi, JHEP 08, 007 (2004), hep-lat / 0306014.

[8] A. Shindler, Phys. Rept. 461, 37 (2008), arXiv: 0707.4093 [hep-lat ].

[9] ETM Collaboration, B. Blossier et al., JHEP 04, 020 (2008), arXiv: 0709.4574 [hep-lat ].

[10] ETM Collaboration, C. Alexandrou et al., Phys. Rev. D78, 014509 (2008), arXiv : 0803.3190 [hep-lat].

[11] ETM Collaboration, K. Jansen, C. Michael and C. Urbach, arXiv:0804.3871 [hep-lat].

[12] R. Frezzotti and G. Rossi, PoS LAT2007, 277 (2007), arXiv:0710.2492 [hep-lat].

[13] G. Colangelo and C. Haefeli, Nucl. Phys. B744, 14 (2006), arXiv: hep-lat/ 0602017.

[14] P. Dimopoulos et al., PoS LAT2007, 241 (2007), arXiv: 0710.0975 [hep-lat].

[15] H. Leutwyler, Nucl. Phys. Proc. Suppl. 94, 108 (2001), arXiv : hep-ph/ 0011049.

[16] G. Colangelo, S. Dürr and C. Haefeli, Nucl. Phys. B721, 136 (2005), hep-lat/ 0503014. 